\title{
Cerebral venous thrombosis in patient of relapse of ulcerative colitis: report of a case
}

\author{
Rajat Agarwal, Anuradha Batra, Ish Anand, Davinder Singh Rana, Samir Patel \\ Department of Neurology, Sir Ganga Ram Hospital, Old Rajinder Nagar, New Delhi 110060, India.
}

Correspondence to: Dr. Rajat Agarwal, Department of Neurology, Sir Ganga Ram Hospital, 1st floor, Old building block, Old Rajinder Nagar, New Delhi 110060, India. E-mail: dr.rajatagr@gmail.com

\begin{abstract}
How to cite this article: Agarwal R, Batra A, Anand I, Rana DS, Patel S. Cerebral venous thrombosis in patient of relapse of ulcerative colitis: report of a case. Neuroimmunol Neuroinflammation 2016;3:243-6.
\end{abstract}

Article history:

Received: 29-03-2016

Accepted: 04-09-2016

Published: 28-10-2016

Key words:

Cerebral venous thrombosis,

ulcerative colitis relapse,

inflammatory bowel disease,

extraintestinal complication

\section{ABSTRACT}

\begin{abstract}
Amongst the various systemic complications of ulcerative colitis, cerebral venous thrombosis (CVT) is an uncommon and serious neurological complication mainly associated during episodes of relapse of ulcerative colitis. CVT is suspected to be a consequence of hypercoagulable state occurring during the disease in genetic predisposed persons. Most patients present with rapid neurological deterioration. This devastating intracranial complication requires immediate medical intervention to avoid potentially life threatening consequences. The outcome is good, provided the disease is diagnosed on time and the treatment is started early. The authors present a patient of CVT, a rare complication seen during relapse of ulcerative colitis.
\end{abstract}

\section{INTRODUCTION}

Ulcerative colitis is an idiopathic chronic inflammatory bowel disease (IBD), which results from a complex relationship of environmental factors and genetic predisposition..$^{[1]}$ Ulcerative colitis (UC) is known to have extra intestinal central nervous system (CNS) complications. CNS features in UC are particularly severe and rarely reported due to their varied presentation. These include cerebrovascular disease, myelopathy and cerebral vasculitis. ${ }^{[2]}$ The two most common thrombotic complications of UC are deep venous thrombosis and pulmonary thromboembolism. ${ }^{[3]}$ IBD accounts for $1-6 \%$ of the total causes of cerebral venous thrombosis. The pathogenesis of CNS manifestations of IBD seems to be related to immune mechanisms or prothrombotic states; however the complete pathogenesis is yet to be elucidated. There are no major differences in clinical or radiological characteristics, prognosis, or treatment between patients of IBD-related cerebral venous thrombosis (CVT) and non-IBD related CVT. ${ }^{[4]}$ 
CVT is a uncommon type of cerebrovascular disease that accounts for $0.5 \%$ of all strokes..$^{[5]}$ It usually presents as headache, seizures, focal neurological deficits, altered consciousness, and papilledema. ${ }^{[6]}$ Due to its varied presentation and low incidence, CVT is not readily suspected, leading to delayed treatment and a poor impact on the prognosis.

We report a case of 39-year-old male, with 10 years long history of ulcerative colitis, who presented with cerebral venous sinus thrombosis following disease relapse. He was managed aggressively and the patient improved.

\section{CASE REPORT}

A 39-year-old young male, known case of ulcerative colitis for last 10 years (treated with tablet mesalamine and steroids), presented with complained of increased frequency of stools and abdominal pain for last 1 month. The stool frequency had increased to 6-8 times in a day, associated with blood and mucus. He also complained of new onset, severe daily headache for last 1 week. Headache was associated with nausea and vomiting. There was no history of photophobia or phonophobia. The evening preceding admission, patient went into a state of confusion and altered behaviour. There was no history of fever or seizure. There was no relevant similar past history and no family history of note.

On physical examination, he was afebrile; pulse was $94 / \mathrm{min}$ and blood pressure was $124 / 70 \mathrm{mmHg}$. There was no lymphadenopathy. On neurological examination, he was drowsy, but arousable and confused. Fundus examination showed signs of early papilledema. There was no limb weakness but the bilateral plantars were extensor response. Rest of neurological examination was within normal limits. Laboratory findings showed hemoglobin of $11.4 \mathrm{gm} \%$; the white blood cell count was slightly elevated at $12,500 / \mathrm{mm}^{3}$ and his serum albumin levels were $4.7 \mathrm{gm} \%$. His erythrocyte sedimentation rate was $46 \mathrm{~mm}$ and C-reactive protein was $116 \mathrm{mg} / \mathrm{L}$. His renal function tests, liver function and serum electrolytes were within normal range.

His contrast enhanced magnetic resonance imaging (MRI) brain revealed altered increased signal intensity in the anterior superficial cortical veins and the superior sagittal sinus on T1 weighted (T1W), T2 weighted (T2W) and fluid attenuated inversion recovery (FLAIR) images suggestive of their thrombosis. The high superficial cortical veins in the left fronto-parietal regions also show altered increased signal intensity on TIRM and T1W images suggestive of their occlusion. Similar altered signal intensity was visualized in the Torcula and the right sided transverse sinus. Also a note was made of a large left sided fronto-temporo-parietal and occipital infarct with hemorrhagic reconversion suggestive of a venous infarct [Figure 1]. Magnetic resonance venography of the brain was suggestive of thrombosis in the superior sagittal sinus and right transverse sinus [Figure 2]. Based on patient's history, clinical features and imaging findings a diagnosis of cerebral venous thrombosis was considered. The patient was managed with conventional heparin, anti oedema measures and other supportive treatment. On further work up for CVT, he was found to be Positive (Heterozygous)

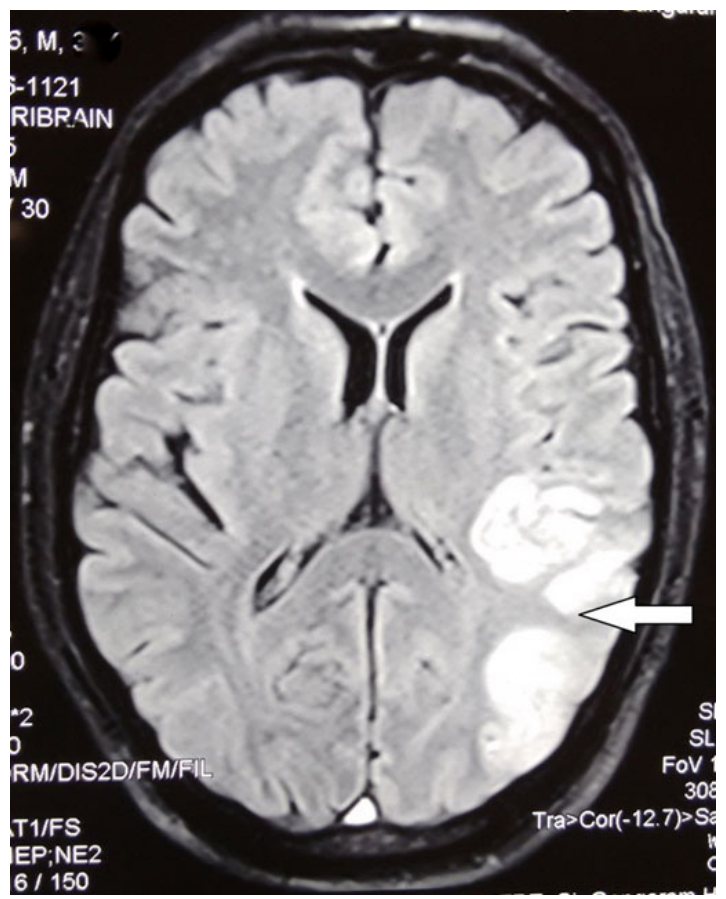

Figure 1: Contrast MRI brain, tirm image: left sided fronto-temporoparietal and occipital infarct. Arrow shows large venous infarct in left parieto occipetal region. MRI: magnetic resonance imaging

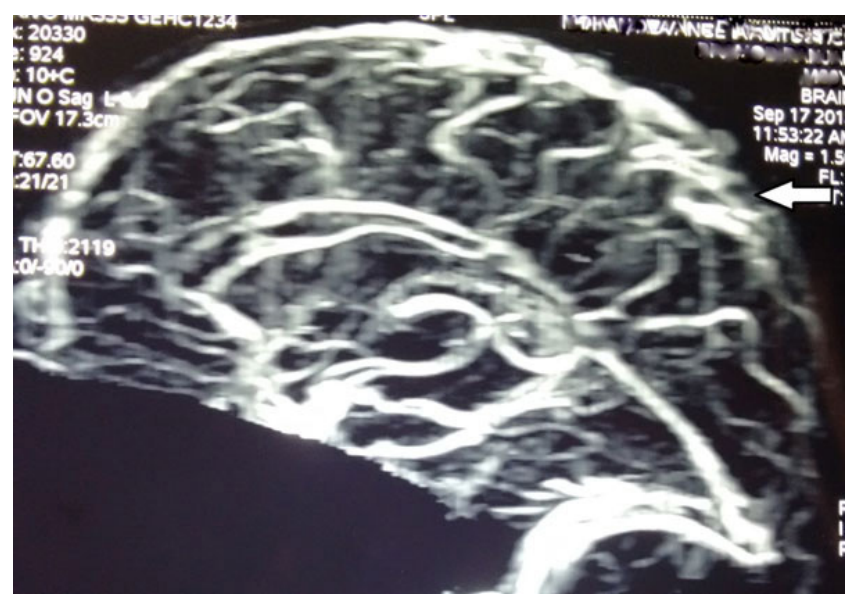

Figure 2: Magnetic resonance venography of the brain showing thrombosis in the superior sagittal sinus and right transverse sinus. Arrow shows occulusion of the superior sagittal sinus 
for MTHFR mutation. Furthermore, he was also found to have serum homocysteine level of $20.5 \mu \mathrm{mol} / \mathrm{L}$. His levels of anti-nuclear antibody, anti-double strand deoxyribonucleic acid, lupus anticoagulants, antiphospholipid antibodies, anticardiolipin antibodies, and anticyclic citrullinated peptides were negative.

The patient clinically improved in a period of five to seven days with the treatment. His symptoms had gradually subsided. He was then discharged.

\section{DISCUSSION}

Extensive thrombosis of venous sinuses is a serious complication of ulcerative colitis. Venous thrombosis is known to occur with a greater frequency than general population in patients with ulcerative colitis. Incidence of thrombosis is about $6.5 \%$ in patients with active IBD. ${ }^{[4]}$ The relationship between thrombosis and UC has not been well defined. But recent evidence suggests that $\mathrm{UC}$ is an important factor for thrombotic complications. ${ }^{[7]}$ The exact cause for the increased rate of thrombotic events in patients with IBD is still uncertain. However, it is most likely related to the interaction between acquired and inherited genetic risk factors. Also, the recent research has suggested it to be an interaction between the coagulation cascade in the body and cytokine mediators of chronic inflammation and also the inflammatory process can itself activate coagulation cascade. ${ }^{[8]}$ The inflammatory process initiates clotting, impairs the fibrinolytic system and decreases the activity of natural anticoagulation mechanisms. Depression of anticoagulation mechanisms not only increases thrombosis, but also potentiates the inflammatory process. That is why, the majority of thrombotic events occur during the active phase of disease. Abnormalities in coagulation cascade such as elevated fibrinogen level, factor V, factor VIII, and increase in circulating thrombin-antithrombin complexes, decreased antithrombin III, thrombocytosis and increased platelet aggregation have been documented. ${ }^{[9]}$ However, there is no significant evidence to associate hematological and coagulation abnormalities with CVT. Grainge et al. ${ }^{[10]}$ analyzed 13,756 patients with IBD and 71,672 matched controls, and found that 139 patients and 165 controls developed venous thromboembolism. Overall, patients with IBD had a 3.4 times higher risk of developing venous thromboembolism than did controls, with risk increasing up to 8.4 times during a flare-up of IBD. Our patient presented with cerebral venous thrombosis, associated with relapse of UC.

MRI brain and a magnetic resonance venography (MRV) should be done immediately, once cerebral vein thrombosis is clinically suspected, so that the early diagnosis is not missed. MRI and MRV are considered the best tools for diagnosis and follow-up. The anticoagulation therapy for patients with CVT is similar in both with active and chronic UC, and has been associated with lower incidence of mortality, if started at appropriate time.

In patients with cerebral venous thrombosis, favourable results are possible with earlier diagnosis and appropriate treatment plan. If patients remain untreated, the mortality rate can be very high. Therefore, the present report highlights the value of considering the diagnosis of CVT, in patients with IBD, especially when the disease is in its relapse phase.

In conclusion, it is necessary to suspect CVT in a patient with relapse of UC, who presents with recent, unusual severe headache, stroke like features, seizures, or any other brain syndrome. The physician, neurologist or the gastroenterologist should be well aware of the increased risk of CVT in patients with relapse of UC. The gastroenterologists treating UC should be wise enough to suspect CVT, especially in genetically predisposed and immediately seek a neurologist's opinion for proper management. Also, it would be useful to investigate for genetic hypercoaguable state in patients of UC, in order to find out the at-risk group of UC patients.

\section{Financial support and sponsorship Nil.}

\section{Conflicts of interest}

There are no conflicts of interest.

\section{Patient consent \\ Patient consent was obtained from the patient.}

\section{Ethics approval}

Ethics approval was obtained prior to the commencement of the study.

\section{REFERENCES}

1. Stam J. Thrombosis of the cerebral veins and sinuses. $N$ Engl J Med 2005;352:1791-8.

2. Scheid R, Teich N. Neurologic manifestations of ulcerative colitis Eur J Neurol 2007;14:483-93.

3. Umit H, Asil T, Celik Y, Tezel A, Dokmeci G, Tuncbilek N, Utku U, Soylu AR. Cerebral sinus thrombosis in patients with inflammatory bowel disease: a case report. World J Gastroenterol 2005;11:5404-7.

4. Twig G, Zandman-Goddard G, Szyper-Kravitz M, Shoenfeld Y. Systemic thromboembolism in inflammatory bowel disease: mechanisms and clinical applications. Ann N Y Acad Sci 2005;1051:166-73.

5. Bousser MG, Ferro JM. Cerebral venous thrombosis: an update Lancet Neurol 2007;6:162-70. 
6. Ameri A, Bousser MG. Cerebral venous thrombosis. Neurol Clin 1992;10:87-111.

7. Quera R, Shanahan F. Thromboembolism -- an important manifestation of inflammatory bowel disease. Am J Gastroenterol 2004;99:1971-3.

8. Danese S, Papa A, Saibeni S, Repici A, Malesci A, Vecchi M. Inflammation and coagulation in inflammatory bowel disease: the clot thickens. Am J Gastroenterol 2007;102:174-86.
9. van Bodegraven AA, Schoorl M, Baak JP, Linskens RK, Bartels PC, Tuynman HA. Hemostatic imbalance in active and quiescent ulcerative colitis. Am J Gastroenterol 2001;96:487-93.

10. Grainge MJ, West J, Card TR. Venous thromboembolism during active disease and remission in inflammatory bowel disease: a cohort study. Lancet 2010;375:657-63. 